\title{
What Angles Can Tell Us About What Holes Are Not
}

\begin{abstract}
In this paper I argue that holes are not objects, but should instead be construed as properties or relations. The argument proceeds by first establishing a claim about angles: that angles are not objects, but properties or relations. It is then argued that holes and angles belong to the same category, on the grounds that they share distinctive existence and identity conditions. This provides an argument in favour of categorizing holes as one categorizes angles. I then argue that a commitment to the existence of properties to be identified with holes provides sufficient resources to account for true claims about holes.
\end{abstract}

\section{Introduction}

In this paper I will argue for a claim about what holes are not: specifically, that holes are not objects. To this extent I will be arguing against a certain kind of realism about holes: one which construes holes as particular objects. If I am coy about the extent of my conclusions, however, this is because an adequate account of what holes are depends first on an adequate account of what the fundamental categories of things are: i.e. an adequate ontology. This is a matter over which there is substantial philosophical disagreement. My purpose here is to provide an argument against this specific kind of realism about holes which is not hostage to the correctness of a particular ontological system.

Before proceeding, however, I should be clear about what I mean by realism about holes. One dispute someone may have with the doctrine that holes are real entities can be 
characterised in terms of a disagreement about whether we should take seriously the existential commitment implicit in sentences about holes featuring existential quantification, as in 'There is a hole in my heart.' On this view the realist thinks there are holes and the non-realist thinks there are not. A dispute framed in such a way immediately puts common sense on the side of the realist, as it requires the non-realist to deny the existence of holes. ${ }^{1}$ However, the feeling that holes are ontologically suspect need not find expression in just this one kind of dispute. An alternative expression of such concerns might be a disagreement about what holes are: a disagreement about which of the fundamental divisions of reality holes fall into. Are holes objects or properties? Are they universals or particulars? It is this dispute that I wish to attend to in this paper: which fundamental ontological category do holes belong to?

Of course, an interesting discussion about which ontological category something belongs to presupposes some level of agreement about what ontological categories there are and, as I have said, this is an issue over which there is substantial disagreement. In the present paper I will restrict the discussion to just one categorial distinction: the object/ property distinction. This will allow me to illustrate some of the implications of my arguments for two influential ontological systems in metaphysics which accept this distinction: the two category ontology favoured by David Armstrong and the four category ontology defended by E J Lowe. My conclusion will be that whatever holes may turn out to be, they will not be objects: so, in Armstrong's ontology they will not be particulars, but universals. In Lowe's ontology they will neither be what he calls 'substances' nor what he calls 'kinds', but will fall into one of the other two fundamental categories.

In what follows I will call the view that holes are objects of some sort 'objectualism', and the contrasting view that holes are properties or relations of some sort 'non-

\footnotetext{
${ }^{1}$ More will be said on this view later. See section 4 .
} 
objectualism'. So, my argument is intended to show that objectualism about holes, in this sense, is mistaken. In this way, the kind of realist position which categorises holes as particular, immaterial objects which are the complements of material objects, such as that elaborated by Casati and Varzi in their classic discussion of the topic, Holes and other superficialities, will be ruled out of court. However, it will also rule out the (also realist) view of holes as particular material objects of some sort, as defended by the character of Argle in David and Stephanie Lewis' dialogue on holes. In spite of this latter point, the view I articulate here is closer in spirit to this 'Ludovician' account of holes, as Castai and Varzi call it, though not on account of any particularly strong sympathy with materialist or more general naturalist tendencies.

My strategy for arguing for this conclusion proceeds by way of an argument about the nature of angles. The rationale for this strategy is that angles bear many of the distinctive, puzzling features of holes, so a sound understanding of what kind of thing an angle is should give us some indication of what should be said about holes. The argument presented in this paper has three stages. The first stage consists of an argument for the claim that angles should not be categorised as objects or particulars, but rather as relations. The second stage argues that the categorisation of holes should be the same as the categorisation of angles. The upshot of these two sections will be the claim that holes should not be categorised as objects. The final stage of the argument will be to say something about the quite general challenge to the 'non-objectualist' to outline a schema for expressing all true sentences about holes in a way that does not imply the existence of objects that are the holes.

\section{Angles are not objects}


The first stage of the argument is to say something about what kind of entity an angle is. So, what kind of an entity is an angle? In particular, is it an object or a property or relation? Before answering this question, it is important to provide some definitions of these philosophical terms of art. I have said that I intend my argument to not be hostage to a particular ontology, so my discussion will involve considering a number of ways of capturing the distinction in question. The first thing to stipulate is that where I talk about properties, I intend this to include relations, so the distinction in question is between objects on the one hand and properties and relations on the other. One characterization of this distinction is that objects are essentially bearers of properties. This characterization says almost nothing about the nature of properties, though, and this means we must give a more detailed characterization of properties. One characterization, introduced by Jerrold Levinson, is that properties are ways of being: ways things are. ${ }^{2}$ This idea that properties are ways of being is minimally what I require for my argument in favour of non-objectualism about holes, but it is worth observing that the object/property distinction, if it is to be exclusive and exhaustive must be such that we can say that anything that is a way of being cannot be an object. This is something Lowe captures in his characterization of the distinction, where objects are construed as entities of order zero and properties are understood as entities of an order greater than zero. The general idea is that entities of order greater than zero characterize those entities of a lower order, but those of order zero characterize no entity at all - an idea that captures the traditional Aristotelian notion of a substance. ${ }^{3}$

I will briefly also say something about Armstrong's treatment of the object/property distinction. For Armstrong the object/property distinction is taken to be adequately

\footnotetext{
${ }^{2}$ See Levinson (1978)

${ }^{3}$ See Lowe (2006), p. 72
} 
captured by the particular/universal distinction, which provides an explanation of how nonidentical things can have the same property, or have the same way of being. If properties are construed as Armstrong's universals, then this does require the 'non-objectualist' position to be slightly more complex than if we admit particular properties, but I will address this in the third section of my discussion. What is important here is that, for Armstrong, universals are supposed to stand to particulars as properties are thought to stand to objects: as ways those objects are. So, with the idea in place that properties are ways objects are, let us turn to the question of whether angles are properties or objects.

We must first distinguish between two different uses of the word 'angle'. Sometimes this word is used in a complex expression that refers to a figure formed by lines or planes, such as in 'this angle' in the sentence 'this angle is made of red lines' ${ }^{4}$ In such cases, the word 'angle' serves to enable ostensive reference to a figure by specifying a property of the object being referred to. However, in so far as the word 'angle' has a reference of its own in this example, its reference is a shape property. Just as we have only a comparatively small number of words to express colour variety compared to the vast number of colour properties, so too are there many shape properties we do not have names for: for instance, the particular shape of my cat as he sits, unmoving on my desk. It is for this reason that the above expression may be used to speak about a wide range of figures. Compare the above expression about an angle with an analogous expression about a shaped figure: 'this square is made of red lines'. Here we have a word that refers to some fairly specific shape property which characterizes the figure in question. In both cases, though, these two claims involve a commitment to an object that is a figure of a certain kind, or is shaped in a certain way.

It seems, then, that one use of 'angle' clearly does carry with it a commitment to objects. However, this is not particularly philosophically interesting, because it does not tell

\footnotetext{
${ }^{4}$ The examples I concentrate on in this section are two dimensional angles, but I discuss three dimensional angles in section 4 .
} 
us whether angles fall on the 'object' side or the 'property' side of the distinction between objects and properties. All that has been made plain so far is that, when they are true, such sentences entail the existence of some object that is a certain way. In the case of 'this square is made of red lines' it is the existence of an object that is square. In the case of 'this angle is made of red lines' it is some object that is shaped in the distinctive way that we get by putting two straight lines together so one of their ends meet. It will be necessary to refer to this distinctive way of being throughout the course of my discussion, so I propose to use the expression 'angular', as in 'some object is angular' and 'this is an angular figure'.

However, there is a second use of the word 'angle' whose ontological implications are much less clear and which is more revealing of the ontological status of angles. This is the use employed when we speak of a particular figure having an angle of a certain measure or when we speak of different figures having the same angle. This second use of 'angle' is the one that is the subject of mathematical investigation and expression: in mathematics an angle between two lines is construed as the amount of rotation about a point of intersection that is required to bring one line into correspondence with another.

What should we say about this second use of 'angle'? One way of addressing this question is to take the syntax of expressions where it occurs as an initial guide to the ontology. So, consider an expression of the form 'figure a has an angle of $\mathrm{n}$ degrees'. In so far as we might be entitled to do so, we could read off from the syntax that three entities are involved in the state of affairs described: the object a, which is a figure; an angle; and a measure of that angle. However, this would not settle the ontological status of the second and third entities in the list. The measure of the angle can be dealt with fairly straightforwardly: being a measure of the angle strongly suggests it is a way of being for the angle, and so falls on the property side of the object/property distinction. The second entity 
in the list, the angle, is not so straightforwardly dealt with: is it an object to which the figure $\mathrm{a}$ is related, or is it a property which the figure bears?

Thus far we have two rival ontologies for states of affairs involving angles: one which counts the angle had by the figure as an object; one which counts it as a property. These rival ontologies were arrived at by considering the syntax of sentences about figures having angles. However, syntax does not always indicate the correct ontology. In the present case, the following consideration is important: whenever there is an angle, there will also be (at least) two lines that are spatially related, which compose the angular figure. This point is significant for two reasons. The first is that it provides an argument against the first ontology specified above, which construes the angle as an object to which the angular figure is related.

How might this ontology incorporate the preceding point? It might be said that the lines compose the angular figure, or that the lines compose the angle. But how are we to decide which they compose? One suggestion might be that the locution ' $x$ has angle $y$ ' should be understood as expressing a relation of constitution between an angular figure and an object, the angle. So, the lines compose the angle, which constitutes the angular figure. The difficulty with this view lies in that fact that the composition conditions for the angular figure and the putatively distinct object that is the angle are just the same. In cases of constitution, such as when a statue is constituted by a lump of bronze, the motivation for counting the lump and the statue as numerically distinct is that the composition conditions for each are different. But this is not the case here: the composition conditions for the angular figure and the putatively non-identical object, the angle, are just given in terms of the lines composing them. Both the angle and the angular figure can survive replacement of the composing lines with other, numerically distinct lines. This sameness of composition 
conditions argues in favor of counting only one of these entities, the angle and the angular figure, as an object.

A second consideration against the present ontology has to do with patterns of existential dependency between the angle and the angular object. The angle depends for its existence on the object: obliterate the object and there will be no way it is. This pattern of dependency is characteristic of the idea that properties are ways objects can be. This thought gets captured in different ways by different ontological systems: Lowe captures this in terms of rigid existential and identity dependence of particular properties on the particular objects they characterize; Armstrong captures this idea, though less fully, in his commitment to immanent realism about universals. Armstrong's position does not capture the full content of the intuition that the existential dependence of properties on objects is rigid, but this is perhaps so much the worse for his ontological system. Irrespective of this, even in Armstrong's ontology this point about the pattern of ontological dependence still favours classing angles on the property side of the object/property distinction.

The second significant feature of the observation that certain lines, which are spatially related in a certain way, compose the angular figure is that it provides reason for thinking that we may have been mistaken in trying to arrive at our ontology simply from the syntax of a particular group of expressions. The syntax of an expression like ' $x$ has an angle of $n$ degrees' suggested three entities; however, the observation that when there is an angular figure there will also be some parts of it which are spatially related suggests a more minimal ontology. We might put the point like this: there is an angular figure which is composed of lines which are angled, where 'angled' is shorthand for some complex predicate expressing a spatial relation between the lines. This account would be ontologically more parsimonious than the ontology which acknowledges an angular figure, a property possessed by it, a property of that property and lines composing the figure and the 
spatial relation between them. However, in this more parsimonious ontology, the angle is just the spatial relation between composing lines: that is the thing which is of $n$ degrees. So, even here the angle falls on the property side of the object/property distinction.

These various arguments all point toward the conclusion that an angle, in this second sense, is a property or relation, rather than an object. There remains the question of whether angles should be understood as universals or particulars, which I will leave open here, but will discuss in brief in section three.

\section{Angles and Holes}

If the foregoing argument is correct, then it indicates how we should understand the metaphysics of holes. Following the work of Casati and Varzi in their Holes and other superficialities, we need to have a general account that unifies the following phenomena: cavities, depressions and tunnels. The reason is that each of these phenomena is a case of some material object being shaped in such a way that it surrounds a region of space which could, in principle, not contain any matter. Our ordinary expressions about such phenomena include substantives which, taken at face value, appear to name entities: 'the cavity'; 'the depression'; 'the tunnel'. These entities are also countable: a material object can have, or host, two cavities, three depressions, four tunnels. Moreover, accounts of these phenomena seem to run up against the same metaphysical puzzles: if we replace some parts of a material object hosting one of these things with other parts of the same stuff, we have a different lump, but the same cavity, depression or tunnel. If we replace parts of the matter filling the space surrounded by the material object with parts of the same original kind, then we still have the same cavity, depression or tunnel. Also, these entities seem to depend upon their host for their existence in some way: destroy the material object and we are not 
left with the cavity, depression, or tunnel. Lewis Carroll's fiction of the Cheshire Cat's grin remaining after the cat had disappeared takes its absurdity from this point.

These points can be summed up in the following way: all three cases share distinctive existence and identity conditions, so they should be all counted as instances of a single sort of phenomena: a hole. In addition, there is a similarity in respect of their epistemic status: perceptual awareness of the entity in all cases depends upon perceptual awareness of the object hosting them. Perceptual awareness of a tunnel depends on perceptual awareness of some portion of its host. This is true also of cavities, although there are contingent reasons why we cannot perceive many cavities, such as the density or thickness of the host. We can perceive some cavities, as when we look at a mosquito net, or some other thin material, hanging draped over a bed. If we had some form of perceptual system utilizing sonar, then we could become perceptually aware of cavities in denser or thicker materials. As with the metaphysical points, this also argues, though less strongly, in favour of a single unified account of these phenomena.

So, the reason for treating cavities, depressions and tunnels as the same kind of entity, for demanding a unified account of these phenomena, is that they give rise to the same metaphysical puzzles. A solution to these puzzles for one of them would be adequate as a solution for all of them. What I will argue now is that exactly the same considerations apply to angles. Consequently, the account of what angles are and the account of what cavities, depressions and tunnels are must be the same.

One thing that is of note about cavities, depressions and tunnels is that when the material object they are hosted by is three dimensional, there will be two dimensional cross sections of that object which are very like angles. Let's start with the simplest case: take a conical depression, such as is found in children's party hats. Now take a two dimensional slice of that conical depression which cuts through the vertex. This will produce a $\mathrm{V}$ shape: 
an angular figure. Whatever kind of thing we have here, it generates all the same metaphysical problems that the three dimensional holes generate:

1. There is some material object shaped in such a way that it surrounds a region of space which could, in principle, not contain any matter.

2. Our ordinary language expressions of such phenomena include substantives which, taken at face value, appear to name entities: e.g. 'the angle'.

3. These entities are also countable: the difference between ' $V$ ' shapes and ' $W$ ' shapes shows up in true statements featuring quantifiers: 'There is one point of intersection', etc.

4. If we replace some parts of the material objects with other parts of the same kind, we have a different lump, but the same angle.

5. If we replace parts of the matter filling the space surrounded by the material object with parts of the same original kind, then we still have the same angle.

6. Also, these entities seem to depend upon their host for their existence in some way: destroy the material object and we are not left with the angle.

7. The perceptual conditions are the same: you see a hole by seeing its 'host' and you see an angle by seeing the thing shaped in a certain way. 
The moral to draw is that whatever general metaphysical story we tell about angles, the same account should be given for holes. However, this applies not simply to those figures arrived at by taking a two dimensional slice of a material object with a depression that has a solid angle in it: it also applies to depressions which are curved. Consider the depression in a soup bowl: if we take a two dimensional slice of this which cuts through the bottom of the bowl, then we get a curved figure. This is not composed, like the angle, of two parts standing in a relation but of many point-like parts standing in relations: relations which are captured by the equation for the particular curve in question. This curved figure generates all the same metaphysical puzzles as cavities, depressions and tunnels.

Once curves are admitted to be the same type of thing as angles we can meet an objection that might be raised about angles and the party hat example. It may be objected that there are many more ways of slicing through an object with a depression in it than just though the vertex of the solid angle. We could cut through a conic depression to get a two dimensional slice that is a curved figure or a circular figure. But, now we can see that the curved figure is just the same kind of phenomena as the angular figure. Moreover, the circular figure is no different either: a circle is just a closed curve.

The examples I have been considering are shapes of constant curvature, but this is not essential to the case. The two dimensional figures of variable curvature which would be obtained from taking a two dimensional slice of the Statue of Liberty would also generate the same issues. Now we can see that neither tunnels nor cavities generate any further considerations that need special attention. Any two dimensional slice through either will give a figure of no significant difference from the figures already discussed.

So, angular, curved and circular figures are just two-dimensional instances of the same phenomena that cavities, depressions and tunnels are. But we already have a principled account for angular figures. There is the angular figure, the lines composing it and 
the spatial relation between them. There is no additional object that is the angle, somehow related to the angular figure. There is just the spatial relation, which is what our talk of angles is really about. It is in virtue of this spatial relation that the lines surround a region of space that could in principle not contain any matter. ${ }^{5}$ The argument in the foregoing discussion leads to the conclusion that this account should be adopted for the three dimensional cases: for cavities, depressions and tunnels. There is the shaped object, which is composed of material parts that are spatially related. There is no additional object - no bearer of properties or entity of order zero - over and above the shaped object and its composing parts. The hole is the spatial relation between parts of the object, in virtue of which those parts surround a region of space that could in principle not contain any matter.

\section{How To Talk About Holes}

I have argued that holes and angles raise the same set of philosophical puzzles, particularly in respect of their identity and existence conditions. For this reason they should be categorized as the same sort of entity. I have also argued that angles are most plausibly construed as falling on the property side of the object/property distinction. However, the following objection might be voiced: if angles raise the same kind of problems as do holes, then the success of the view that holes are objects in dealing with these difficulties argues for the conclusion that angles must also be objects. To meet this objection it needs to be shown that the view that angles and holes are properties can adequately solve these puzzles.

\footnotetext{
${ }^{5}$ Even in the more inflated ontology which acknowledges also the property that is the angle and the measure of it, there is no object in addition to the figure.
} 
I have already discussed how 'non-objectualism' about holes deals with the features of composition, constitution and dependency mentioned in items (4), (5) and (6) in the list given in the previous section. I have also discussed items (1) and (2). The remaining challenge, though, for any 'non-objectualist' about holes is to show how it is possible to deal with sentences featuring quantification over holes and angles. This is one of the points which is most easily accommodated by the view that holes and angles are objects. However, I think that there is a fairly straightforward way that this challenge can be met.

Much of the debate on this topic has concentrated on whether it is possible to adequately paraphrase sentences of the form 'there are $\mathrm{n}$ holes in $\mathrm{x}^{\prime}$ in such a way that does not involve existential quantification over holes, but only over the material host objects. This is usually with a view to defending the account of holes developed by Argle in David and Stephanie Lewis' original paper: the view that holes are a special class of material objects, the 'hole linings' or 'skins' of the material objects. The difficulty begins with the observation that the hosts may not just have multiple holes, but multiple kinds of holes, and that different hosts may have the same number of holes which may be dissimilar in shape. The challenge is to find a translation schema which can do justice to the complexity that needs expressing, but without introducing quantification. One suggestion discussed and dismissed by Casati and Varzi is the use of the sentence schema ' $x$ is ( $n, j, k$ )-holed' to paraphrase sentences of the form 'there are $n(j, k)$-holes in $x^{\prime}$, where $j$ stands for the number of edges of the 'skin' of the hole and k stands for the topological genus of the hole.

Casati and Varzi dismiss this 'adverbial' solution because it is not obvious that this schema can capture the difference between hosts with two unlinked toroidal tunnels and hosts with interlinked toroidal tunnels. This could be dealt with by specifying the location and orientation of the holes, just as we specify the topological genus. In this way some difficulties raised by the solution suggested by Michael Tye, which Casati and Varzi discuss, 
could be avoided. Tye's suggestion was that we express the situation where a host has two interlocking tunnels by quantifying over interlocking empty spatial regions, rather than holes. The situation could then be expressed in terms of the following schema:

There are two interlocking, torus-shaped empty spatial regions $\mathrm{x}$ and $\mathrm{y}$ internal to the host, and the host is $(1,0,1)$-holed at $x$ and $(1,0,1)$-holed at $y$.

Casati and Varzi point out that this solution demands an explanation as to why hosts can only be holed at the empty regions in question.

Whether or not these difficulties can be answered by Argle, I think that this debate can be neatly sidestepped by the view that holes are properties or relations between parts of the host. This debate gets under way because the non-realist is, according to Casati and Varzi, trying to avoid existential quantification over holes, but existential quantification is an easy way of capturing claims about number. As I construe non-objectualism, though, there is no reason to avoid quantification: on my construal the non-objectualist accepts that holes exist, but does not accept that they are objects. So, the non-objectualist just needs to appeal to quantification over properties and this can be done in either of the ontological systems I have been discussing.

It is intelligible to talk about objects having more than one instance of the same property. Consider a ball which has a black line dividing it into two equal hemispherical regions, each of which is uniformly the same shade of red. Here the ball has (at least) two instances of the same colour. Precisely how we should capture this depends on which ontological system we find most compelling, but in respect of the two ontological systems I have been discussing here there is no serious difficulty in capturing this thought in a way that allows us to count either colours or holes. According to Lowe's ontology, the account 
would be that the host is characterized by two numerically distinct particular colour properties, or modes, which are each instances of the single colour universal 'red'. The same account can then straightforwardly be extended to holes and angles: an object can be characterized by multiple properties, which are the holes and which are instances of a universal.

In the case of Armstrong's ontology, things are slightly more complex. In the case of the ball, the universal 'red' is instantiated in two places at the same time, because it is wholly present in two places at the same time. Nonetheless, on Armstrong's view, strictly speaking there is just one universal. However, it is not open to Armstrong to say that there is one particular that stands in two numerically distinct instantiation relations to the universal 'red'. To say this would open the door to an infinite regress in accounting for the instantiation relation. To avoid this problem Armstrong construes the instantiation of the universal by a particular as involving 'no addition of being'. If this is so, then we cannot consider 'instantiations', in this sense, as countable entities.

This difficulty for the two-category ontology can be dealt with by observing that in the case of the ball there are two numerically distinct particulars that separately instantiate the universal: the two halves of the ball. These halves are component parts of the ball. This solution can be applied to the case of holes and angles in the following manner: consider a particular angular figure like ' $\mathrm{V}$ ', composed of two lines. The angle is the relation between the two lines: in Armstrong's ontology we say that the pair of lines instantiate the relational universal: the relational universal is the angle. Now, consider an angular figure like 'I_l' with two angles. Here the figure is composed of three lines, but each angle can remain instantiated even when one of the lines is removed - either the left upright or the right upright line. If we remove an upright, say the right one, we are left with a component part of the original figure, but which has just one angle: the relation between the parts. If, on the 
other hand, we were to remove the left upright from the original figure we would also be left with a component part with just one angle: the relation between its parts. However, each of these component parts of the original figure has some part in common: the horizontal line. So, in Armstrong's ontology we can still truly say that there are two instances of the same property, because there are two distinct particulars that instantiate the same relation. However, on this view, these particulars are not the angles themselves, but angular figures.

However, there remain some true claims that can be made about holes that will need paraphrasing by the non-objectualist. Consider these two sentences:

1. The right ventricle of his heart has enlarged.

2. We have passed a catheter through the hole in his heart.

The first sentence expresses a case of numerical identity over time, with a qualitative change in one cavity of a heart. This implies an object, the cavity, changing over time. The problem for the non-objectualist is that it is not immediately clear that they have the resources to give a plausible account of such cases. One strategy that is available to the nonobjectualist to deal with them is to observe that (1) will be true in one of these two situations:

(a) The relations between the parts of the heart wall are changed (the heart walls become stretched) or 
(b) some parts of the heart wall are removed to produce a heart wall made of some of the remaining parts of the heart wall (the heart walls are thinned).

They could then suggest that (1) is simply a paraphrase of either (a) or (b). Neither (a) nor (b) would require the introduction of an object that is the cavity: they would both require the introduction of other objects, such as the parts of the heart wall. Consequently, (1) doesn't require the introduction of an object that is the cavity. There is a cost to this move, though: because the change takes place over time the original relation which the nonobjectualist identifies with the ventricle must cease to be instantiated - so the nonobjectualist may be committed to no identity over time for holes.

Sentences like (2) are tricky for a different reason. It is not possible to pass anything through properties, whether they are conceived as universals or particulars. Nonetheless, this can be dealt with as follows. When a sentence like (2) is true, something will have been passed through the region of space bounded by the parts of the heart that instantiate the relation that is the hole. So, claims about interaction with holes like (2) can be understood as paraphrases of sentences mentioning the region of space which the hole marks out.

\section{Conclusion}

In their 2004 paper 'Counting the holes', Casati and Varzi argue that if you have two different languages which are equally good for expressing things about holes, but one employs substantives like 'the hole' and the other makes no such use, then nothing can be settled about the metaphysics of holes by appeal to the expressive power of the language. Each language entitles us to hold different metaphysical views about holes, but the expressive power of the language can decide nothing about the correctness or incorrectness of any such metaphysical position. An argument of a different sort is needed if we are to 
decide on the adequacy of a metaphysics of holes. In this paper I have tried to articulate such an argument with the following structure:

1. Angles are not objects but are properties or relations.

2. Whatever angles are and are not, holes will also be and not be.

3. So, holes are not objects, but are properties or relations.

At the outset of the Lewis" original discussion of holes, Argle declares "I believe in nothing but concrete material objects". Bargle then raises the existence of holes as a difficulty for Argle's nominalism. The Lewises were correct to address this challenge: holes are perplexing entities precisely because they raise problems about where to fit them into any ontology. Those, like David Lewis, whose concern for ontological economy leads them to limit the number of ontological categories face difficulties in preserving the truth of many sentences about holes. Other philosophers, such as Casati and Varzi, take such difficulties to show the need for a more inflated ontology than Lewis' demand for ontological economy would permit. However, even philosophers who agree with Casati and Varzi about this find their introduction of a sui generis entity, such as an immaterial object, difficult to accept probably because such objects are not acknowledged by any physical science. ${ }^{6}$ However, the suggestion I have outlined here, that holes are relations, offers the twin advantages of dealing with the many sentences about holes that a more limited ontology cannot, and of doing so by placing holes in a category that, plausibly, is required by the physical sciences. This is because the categorical distinction between object and property is introduced to give an adequate account of states of affairs, which it is argued are needed for truthmaking. This is not to insist on the correctness of this point of view, but just to point out that, if correct,

\footnotetext{
${ }^{6}$ See, for example, Wake, Spencer, Fowler (2007)
} 
would forestall the kind of reservations that are entertained about Casati and Varzi's account of holes.

At the outset of my own discussion, I observed that any attempt to say which category holes belong to presupposes some prior commitment about what the fundamental ontological categories are. Instead, I have laid out the ways in which my claim that holes are relations can be developed within two different ontological systems which both acknowledge a fundamental categorical distinction between objects and properties. In view of the difficulties holes raise for Lewis' nominalism, this provides some measure of support for these ontologies over Lewis'. However, as we have seen, the two category ontology favoured by Armstrong runs into some difficulties over counting instantiations which are not encountered by the four category ontology favoured by Lowe. This provides some reason to prefer the latter ontological system over the former; though this reason is, of course, defeasible.

There is one final point of interest with the position I have defended which is worth noting here: recently Roy Sorensen ${ }^{7}$ has defended the view that shadows are holes in light and that the perceptibility of such absences means we should construe them as causally efficacious. However, construing holes as relations, as I have suggested here, may provide a way of preserving Sorensen's plausible account of the metaphysics of holes without a commitment to the idea that their perceptibility requires acknowledging causally efficacious absences.

\section{Bibliography}

\footnotetext{
${ }^{7}$ See Sorensen (2008)
} 
Armstrong, D. M. 1974. Infinite regress arguments and the problem of universals, Australasian Journal of Philosophy, 52/3: 191-201

Armstrong, D. M. 1997. A world of states of affairs, Cambridge: Cambridge University Press

Casati R. and Achille Varzi 2004. Counting the Holes, Australasian Journal of Philosophy, 82: 23-27

Casati R. and Achille Varzi 1995. Holes and Other Superficialities, Cambridge: MIT Press

Levinson, Jerrold 1978. Properties and Related Entities, Philosophy and Phenomenological Research, 39/1: 1-22

Lewis, David and Stephanie Lewis 1983. Holes in Philosophical Papers Volume I, ed. David Lewis, Oxford: Oxford University Press: 3-9

Lewis, David 1996. Review of Holes and Other Superficialities by Roberto Casati and Achille C. Varzi, The Philosophical Review, 105/1: 77-79

Lowe, E. J. 2006. The Four-Category Ontology: A Metaphysical Foundation for Natural Science, Oxford: Oxford University Press

Sorensen, R. 2008. Seeing Dark Things: The Philosophy of Shadows, Oxford: Oxford University Press 
Wake, A.; Spencer, J. and Fowler, G. 2007. Holes as Regions of Spacetime, The Monist, 90/3:372-378 